\title{
Estudo da separação entre pistas de pouso e decolagem e de taxiamento em aeródromos brasileiros - aplicação ao Aeroporto Internacional de Fortaleza
}

\author{
Francisco Heber Lacerda de Oliveira ${ }^{1}$, Renan Santos Maia ${ }^{2}$, Gledson Silva Mesquita Júnior ${ }^{3}$, \\ Wilkens Martins dos Santos ${ }^{4}$
}

1Universidade Federal do Ceará, heber@det.ufc.br

2Universidade Federal do Ceará, renanmaia@det.ufc.br

3Universidade Federal do Ceará, gledson@det.ufc.br

4Fraport Brasil - Fortaleza Airport, w.santos@fraport-brasil.com

\section{Recebido:}

12 de janeiro de 2019

Aceito para publicação:

26 de julho de 2019

Publicado:

30 de abril de 2020

Editor de área:

Alexandre de Barros

\section{Palavras-chaves:}

Segurança operacional,

Análise de risco,

Taxiways paralelas,

Colisão.

\section{Keywords:}

Safety,

Risk analysis,

Parallel taxiways,

Collision.

DOI:10.14295/transportes.v28i1.1934

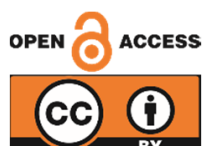

\begin{abstract}
RESUMO
Inadequações relativas ao projeto geométrico podem ser consideradas comuns em aeroportos pelo mundo. No Brasil, alguns aeroportos apresentam inconformidades de separação entre eixos de pistas de pouso e decolagem e de taxiamento paralelas que são inferiores aos requisitos exigidos pela Agência Nacional de Aviação Civil. Este trabalho objetiva avaliar o impacto de tal situação no risco de colisões associado a operações simultâneas de aeronaves em condições de operação por instrumentos. Para tanto, foi utilizado um método de análise de risco proposto pelo Airport Cooperative Research Program do Transportation Research Board com aeronaves de três categorias distintas que operam no Aeroporto Internacional de Fortaleza. Percebeu-se que há probabilidades reduzidas de acidentes acontecerem nestas condições, sendo o método apresentado um meio relevante para a análise quantitativa dos riscos operacionais em tais situações de inconformidade, podendo esta abordagem ser aplicada em casos similares.
\end{abstract}

\begin{abstract}
Deviations from geometric design standards are common in airports around the world. In several Brazilian Airports, there are non-conformities in distances between parallel runways and taxiways, which are lower than the requirements set by the National Civil Aviation Agency. This paper aims to assess the impact of such deviations on the risk of collisions associated with simultaneous operations on the airport site, especially in the case of instruments' operation. To do so, a risk analysis method proposed by the Airport Cooperative Research Program of the Transportation Research Board was applied with three different aircraft types that operate at Fortaleza International Airport. It was found that there is a low probability of accidents and that the method used is an effective way to quantify the operational risk of lower-than-standard separations between parallels runway and taxiways, being this approach useful for similar situations.
\end{abstract}

\section{INTRODUCÃO}

A infraestrutura aeroportuária brasileira vem sendo desenvolvida na última década para atender às exigências de segurança operacional de aeronaves mais modernas e de maior porte. Diversas adequações do lado ar foram conduzidas pelos operadores aeroportuários de modo a cumprir os requisitos estabelecidos pelos regulamentos nacionais e internacionais. ICAO (2018) e ANAC (2018) guiam os padrões para o projeto geométrico de aeroportos no Brasil, 
cujo fim principal está em oferecer condições seguras às operações de pousos, decolagens e taxiamento. Dentre esses padrões, incluem-se as separações espaciais entre as pistas de pouso e decolagem (runways - RWY) e as pistas de taxiamento (taxiways - TWY), além de equipamentos de navegação aérea e outros objetos ou obstáculos presentes na faixa de pistas.

Aeroportos mais antigos datam de períodos cujos padrões de projeto foram planejados para o atendimento de aeronaves menores; nestes casos, é comum a ocorrência de separação entre pistas em desacordo com as exigências atuais. Com o passar do tempo, as operações de aeronaves de maior porte impuseram a necessidade de modificações da infraestrutura e da legislação existentes para a sua acomodação. Aplica-se esse fato ao caso do Aeroporto Internacional de Fortaleza, construído em 1943, para apoio a operações militares durante a II Guerra Mundial, e que hoje opera aeronaves de categoria E.

Adequações de separação entre pistas, muitas vezes, são economicamente inviáveis devido à necessidade de custos elevados para sua implementação ou pela existência de barreiras físicas no entorno dos sítios aeroportuários, sobretudo em áreas densamente urbanizadas. Ainda assim, para que tenham a certificação operacional por parte das autoridades aeronáuticas, os aeroportos brasileiros devem demonstrar conformidade com os requisitos de ANAC (2015 e 2018). Dentre as normas vigentes para projetos de aeródromos, existem dois conjuntos que são frequentemente utilizados: as práticas recomendadas pela International Civil Aviation Organization (ICAO), destinadas a aeroportos internacionais de países membros, e as circulares consultivas da Federal Aviation Administration (FAA), utilizadas, em geral, em aeroportos domésticos estadunidenses. ICAO (2018) é o documento normativo principal, e ANAC (2018) apresenta um conteúdo similar aplicado a aeródromos brasileiros. FAA (2012) é o documento básico e apresenta considerações importantes acerca da segurança e eficiência operacional.

Quanto à separação entre eixos de RWY e TWY paralelas, segundo ICAO (2018), deve-se levar em consideração os seguintes fatores, de modo a proteger a área de segurança das pistas e a zona livre de obstáculos/objetos das faixas de pista: (i) possibilidade das aeronaves aguardarem entre a RWY e a TWY; (ii) interferência sobre o ILS - Instrument Landing System (sistema de pouso por instrumentos para operações de visibilidade reduzida); (iii) existência de TWY de saída rápida; e (iv) possibilidade de as aeronaves manobrarem entre a RWY e a TWY paralela. ICAO (2018), ainda, preconiza que um estudo aeronáutico deve ser conduzido quando não for técnica e operacionalmente possível atender aos requisitos recomendados.

Dessa forma, o presente artigo propõe quantificar, a partir de um método desenvolvido pelo Transportation Research Board / Airport Cooperative Research Program (ACRP Report 051), o risco de colisões associado às operações simultâneas de aeronaves em condições meteorológicas por instrumentos, entre pistas de pouso e decolagem e de taxiamento paralelas. Tal perspectiva busca avaliar a viabilidade de operações com distâncias de separação menores do que o previsto nos regulamentos brasileiros e seu impacto na segurança e na regularidade das operações de aeronaves, como é o caso do Aeroporto Internacional de Fortaleza, estado do Ceará. Assim, o estudo aqui apresentado servirá para avaliar se o desvio observado não afetará negativamente a segurança operacional, nem reduzirá de modo significativo a regularidade das operações. Além disso, pode-se refletir quanto à aplicabilidade do método utilizado em casos similares em outros aeroportos brasileiros. 


\section{EXEMPLOS DE AEROPORTOS NO EXTERIOR COM SEPARAÇÃO ENTRE RWY E TWY INFERIOR À RECOMENDAÇÃO DA ICAO}

Alguns aeroportos internacionais de grande porte, em países como EUA, Portugal e Alemanha, possuem condições de operação de pousos e decolagens que apresentam distanciamento entre eixos de RWY e TWY paralelas inferiores ao mínimo recomendado pela ICAO, no Anexo 14, datado do ano de 2018, conforme exposto na Figura 1.

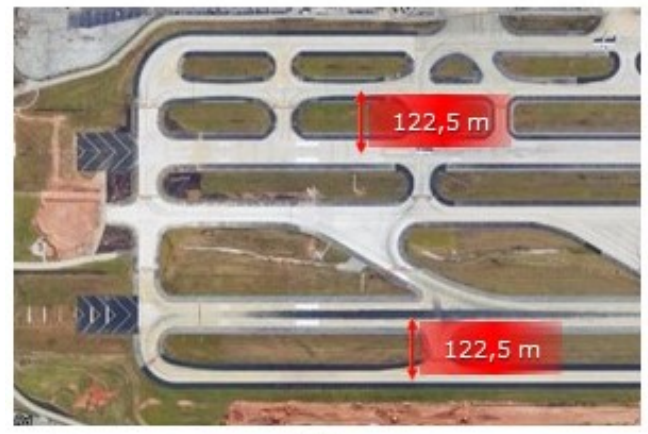

(a)

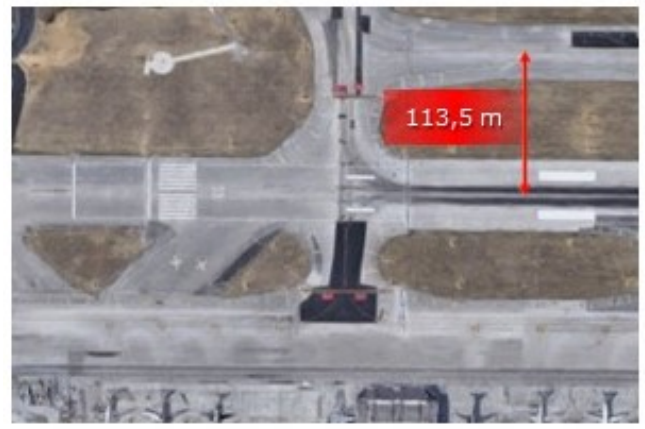

(c)

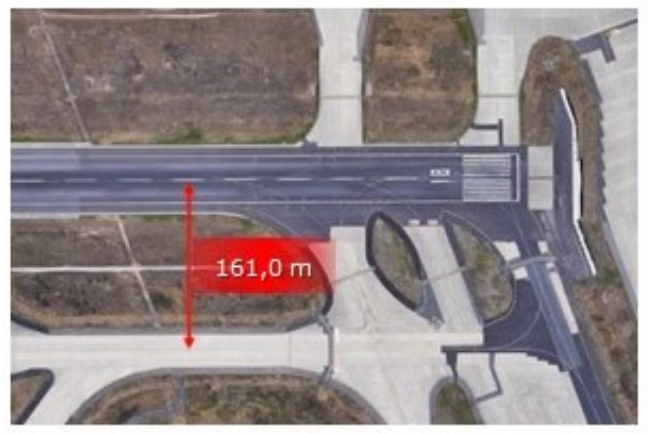

(e)

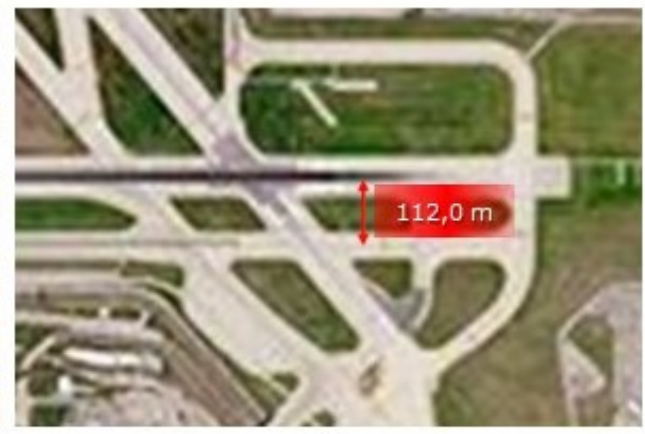

(b)

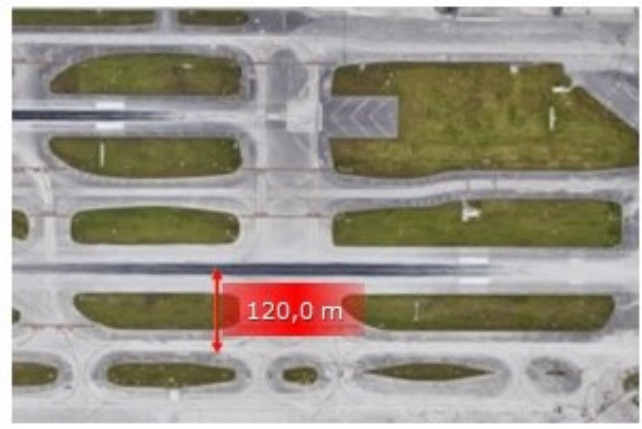

(d)

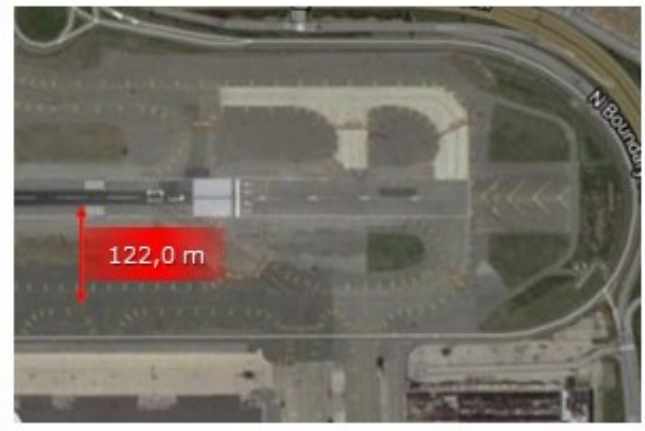

(f)

Figura 1. Distâncias entre os eixos de RWY e TWY paralelas em diferentes aeroportos. (a) Hartsfield Jackson Atlanta International Airport - RWY 9L/9R (Atlanta, EUA); (b) O’Hare International Airport - RWY 27R/32L (Chicago, EUA); (c) Aeroporto de Lisboa - RWY 35 (Lisboa, Portugal)*; (d) Miami International Airport - RWY 26R/26L (Miami, EUA); (e) Frankfurt Airport - RWY 18 (Frankfurt, Alemanha)*; e (f) J.F.K. International Airport - RWY 13L (New York, EUA). *Pistas com restrições para operação

Para aeródromos de Código 4, ou seja, com comprimento básico de RWY maior ou igual a 1.800 m, as distâncias mínimas de separação entre eixos de RWY e TWY paralelas, que operam por instrumentos, são, segundo ICAO (2018), de 172,5 m para aeronaves de Código E (aquelas com envergadura maior ou igual a $52 \mathrm{~m}$ e menor que $65 \mathrm{~m}$ ) e de $180 \mathrm{~m}$ para as de Código F 
(envergadura maior ou igual a $65 \mathrm{~m}$ e menor que $80 \mathrm{~m}$ ), as duas categorias de aeronaves de maior porte em operação na atualidade. Para aeroportos brasileiros, ANAC (2018) indica que essas distâncias são, respectivamente, de 172,5 m e 180 m, em concordância com o proposto em ICAO (2018). Para a última década, não foram encontrados relatos de operadores aeroportuários, autoridades aeronáuticas ou empresas aéreas que mencionem ocorrências de incidentes ou acidentes ocasionados pelo não cumprimento das distâncias mínimas de separação entre as pistas nesses aeroportos.

\section{ESTUDOS DE SEPARAÇÃO ENTRE PISTAS}

A separação entre eixos de RWY, TWY e objetos presentes nas faixas de pistas dos aeródromos é uma questão tratada com atenção há anos pelos órgãos reguladores da aviação civil. Estudos prévios para o desenvolvimento e operação de aeronaves de grande porte nos aeroportos ao redor do mundo destacam tal questão. Travers (1995) expôs a separação entre duas RWY e entre RWY e objetos como possíveis entraves ao desenvolvimento de uma aeronave de grande porte que, à época, fosse capaz de substituir o Boeing 747-400, chegando a ressaltar a necessidade de produção de asas reclináveis como uma solução para evitar tais riscos. Os problemas de separação entre pistas são apenas uma das faces da problemática referente à existência de operações em situações de conflito.

A lógica original da ICAO, de acordo com Hall Jr. et al. (2011), indica que TWY paralelas à RWY devem ser posicionadas de modo a impedir que a aeronave com maior envergadura (aeronave crítica em operação) não penetre na faixa de pista adjacente. Intenciona-se, assim, acomodar eventuais desvios em relação às linhas de eixo nas operações de pouso e decolagem e de taxiamento das aeronaves, obedecendo-se à Equação 1.

$$
\mathrm{S}_{\mathrm{RWY}-\mathrm{TWY}}=\frac{\mathrm{sW}}{2}+\frac{\mathrm{WS}}{2}
$$

onde: $\quad$ SRWY-TwY: distância entre os eixos da RWY e da TWY paralela;

SW: $\quad$ faixa de pista;

WS: $\quad$ envergadura de asa da aeronave crítica.

A avaliação da tendência de desvios foi efetuada por Hall Jr. et al. (2011) e envolve diversos fatores: humanos, meteorológicos, de infraestrutura, falhas de aeronave, dentre outros. Tais componentes podem ser incorporados em uma análise funcional de risco, processo que pode ser aplicado na análise e no gerenciamento de riscos em inconformidades de separações entre diferentes componentes de aeródromos. De maneira geral, percebe-se uma incipiência em relação a esses estudos cujo foco principal consista, especificamente, nas separações entre RWY e TWY. Estudos correlatos efetuados em aeródromos de alguns países podem servir de referência para o entendimento dos princípios que conduzem a riscos na interface RWY-TWY.

Ayres Jr. et al. (2013) avaliaram cerca de 260.000 dados disponibilizados por autoridades da aviação civil de 11 países entre os anos de 1978 e 2012 . Foram investigados acidentes e incidentes ocorridos com a finalidade de analisar quais são os fatores de risco em RWY e áreas operacionais. Para tanto, foram aplicados modelos de frequência, localização e consequência. Os modelos indicaram ser um bom meio para definir diretrizes de projetos aeroportuários. No entanto, foi apontada a necessidade de se atribuir fatores de causalidade aos modelos de localização, uma vez que, por exemplo, as condições meteorológicas podem aumentar ou diminuir o fator de risco. 
Huang (2018) conduziu um estudo para avaliação da sensibilidade de fatores no risco de acidentes oriundos de desvios na trajetória da aeronave após o toque em pista (veer-off). Para isso, utilizou-se o software RSARA2, a partir de dados dos aeroportos internacionais de Hong Kong (HKG), Singapura (SIN) e Nice (NCE). Ainda segundo o autor, a separação entre o eixo da RWY e objetos como uma aeronave taxiando não afetam a probabilidade de ocorrência de desvios, porém afetam na localização, que está diretamente ligada ao comprometimento de segurança que um possível desvio pode representar, quando combinado com outros fatores, como o regime de ventos.

Os métodos existentes na literatura que cumprem a finalidade de avaliação de riscos relacionados a desvios de trajetória de aeronaves e à separação entre pistas incluem os estudos desenvolvidos no âmbito do ACRP (TRB), dentre os quais estão Hall Jr. et al. (2008), Hall Jr. et al. (2011) e Ayres Jr. et al. (2014). A Tabela 1 apresenta um panorama geral do que foi observado por Hall Jr. et al. (2011) para seis casos específicos de aeroportos nas cidades de Addison, Bridgeport, Barnstable, Laconia, Seattle-Tacoma e Taunton. 0 nível de segurança alvo (ou TLS - Target Level of Safety) foi definido em 1 x 10-7, ou seja, uma ocorrência para cada 10 milhões de operações.

Tabela 1 - Resultados da aplicação do método de análise de risco proposto por Hall Jr. et al. (2011) para separação entre RWY e TWY em aeroportos norte-americanos

\begin{tabular}{|c|c|c|c|c|c|c|}
\hline Aeroporto & $\begin{array}{l}\text { Separação - } \\
\text { Norma (pés) }\end{array}$ & $\begin{array}{l}\text { Separação - } \\
\text { Real (pés) }\end{array}$ & $\begin{array}{l}\text { ADG* - } \\
\text { Aeronave } \\
\text { Crítica }\end{array}$ & $\begin{array}{c}\text { Risco } \\
\text { associado ao } \\
\text { pouso } \\
\text { arremetido }\end{array}$ & $\begin{array}{l}\text { Nível de } \\
\text { segurança } \\
\text { alvo (TLS**) }\end{array}$ & $\begin{array}{l}\text { Classificação } \\
\text { de Risco }\end{array}$ \\
\hline Addison Airport (ADS) & 400 & 300 & III & $1,10 \mathrm{E}-09$ & $1,00 E-07$ & Médio \\
\hline $\begin{array}{l}\text { Igor I. Sikorsky Memorial } \\
\text { Airport - Bridgeport } \\
\text { (BDR) }\end{array}$ & 300 & 268 & II & $1,10 \mathrm{E}-10$ & $1,00 \mathrm{E}-07$ & Médio \\
\hline $\begin{array}{l}\text { Barnstable Municipal } \\
\text { Airport (HYA) }\end{array}$ & 400 & 300 & III & $1,10 E-09$ & $1,00 E-07$ & Médio \\
\hline $\begin{array}{l}\text { Laconia Municipal Airport } \\
\text { (LCI) }\end{array}$ & 400 & 210 & III & 5,00E-09 & $1,00 E-07$ & Médio \\
\hline $\begin{array}{l}\text { Seattle-Tacoma } \\
\text { International Airport } \\
\text { (SEA) }\end{array}$ & 500 & 400 & VI & $1,50 \mathrm{E}-06$ & $1,00 \mathrm{E}-07$ & Elevado \\
\hline $\begin{array}{l}\text { Taunton Municipal } \\
\text { Airport (TAN) }\end{array}$ & 240 & 147 & II & $3,00 \mathrm{E}-10$ & 1,00E-07 & Médio \\
\hline
\end{tabular}

O caso mais crítico, conforme se constata na Tabela 1, foi encontrado para o Aeroporto Internacional de Seattle-Tacoma. Com o início da operação do Boeing 747-800, o ADG, parâmetro relacionado ao porte da aeronave crítica a operar no aeroporto, passou da classificação V para VI, elevando os requisitos de separação e proporcionando um conflito com o projeto geométrico do aeroporto. Implicou-se, assim, em um elevado risco de colisão nas operações de aeronaves mais críticas durante os procedimentos em condições meteorológicas instrumentadas de uma ocorrência para cada um milhão de operações.

A concepção de um estudo de risco para aeroportos requer a elaboração de uma estimativa probabilística do nível de risco para a situação de estudo. Posteriormente, deve-se estabelecer um critério para a sua aceitação. Ketabdari, Giustozzi e Crispino (2017) utilizaram na análise de risco o software RSARA (Runway Safety Area Risk Assessment), que utiliza como base os modelos do ACRP (TRB). Além disso, foi feita uma análise de sensibilidade em relação às variáveis que 
foram consideradas no modelo probabilístico. Similarmente, Fernandes, Müller e Alves (2018) aplicaram o modelo de análise de risco ACRP (TRB) contido no Report 050 em um estudo direcionado ao Aeroporto de Joinville. Nesse estudo, o modelo foi utilizado para se calcular o nível de segurança da faixa de pista do aeroporto, sendo apontada também uma necessidade de se elaborar um modelo que fosse adequado à realidade brasileira.

0 método apresentado nos relatórios divulgados pelo ACRP (TRB) se baseia em um chamado Collision Risk Model (CRM), que, a partir da utilização de software de análise estatística, combina probabilidades de deslocamentos verticais e horizontais em relação às trajetórias normais da aeronave, estimando-se, assim, a probabilidade das coordenadas calculadas coincidirem com as do objeto presente nas proximidades da pista analisada, o que representaria, assim, a ocorrência de uma colisão. Para a aplicação deste método, foram analisados CRM para situações comuns e diferentes categorias de aeronaves. Cabe destacar que a estimativa de risco nesse caso é considerada conservadora, dado que: (i) apenas o maior risco ao longo do comprimento da RWY foi utilizado para a estimativa de risco de colisão; (ii) a maior altura de cauda das aeronaves do grupo foi utilizada para representar a aeronave taxiando como obstáculo; (iii) a maior envergadura das aeronaves do grupo foi utilizada para representar a dimensão da aeronave que se aproxima; (iv) a aeronave taxiando foi considerada como sendo um ponto fixo contínuo paralelo à RWY; e (v) a taxa de aproximação perdida de 1\% foi utilizada, enquanto dados do FAA indicam que uma taxa de 0,2\% já é considerada conservadora (Hall Jr. et al., 2011).

\section{APLICAÇÃO DO MÉTODO CONTIDO NOS ACRP REPORTS 051 E 107}

0 método a ser utilizado, apresentado no ACRP Report 051, por Hall Jr. et al. (2011), destina-se a casos em que os projetos para separação entre RWY-TWY, TWY-TWY, RWY-Objetos e TWY-Objetos não podem ser cumpridos. Trata-se de um método útil para avaliar os riscos associados com as separações fora do padrão em aeroportos com restrições existentes e futuras adequações. Os fundamentos de análise se baseiam em desvios verticais (no ar) e laterais aleatórios ocorridos durante operações normais de voos e incidentes veer-off. 0 risco de colisão está relacionado com a probabilidade de grandes desvios da trajetória normal do voo durante o seu trajeto na RWY e na TWY quando existem aeronaves se movendo em rotas paralelas.

Para o cálculo do risco de colisão de aeronaves utilizando simultaneamente uma RWY e uma TWY paralela, consideram-se dois cenários: pouso e decolagem. A análise das operações de pouso divide-se em duas fases: aproximação final e corrida de pouso. Na maioria dos casos, como a maioria dos aeroportos brasileiros possui uma única RWY, ela é utilizada tanto para pouso como para decolagem. A análise para operações de decolagem normalmente não é necessária porque o risco de grandes desvios laterais durante a decolagem é inferior ao risco durante o pouso. No entanto, para este artigo, serão apresentadas as estimativas para os dois tipos de operações.

Matematicamente, o risco associado à ocorrência de eventos de veer-off se dá pelo produto de um modelo básico, que calcula a probabilidade de ocorrência de acidentes dentro das condições operacionais determinadas pelo usuário, por um modelo de localização, que fornece a probabilidade de a distância de ver-off da aeronave ser maior do que um valor de referência. Os modelos estão representados, respectivamente, pelas Equações 2 e 3. Tais equações são customizadas de acordo com a operação realizada: (i) pouso - landing veer-off (LDVO) e (ii) decolagem - takeoff veer-off (TOVO), cujos parâmetros de ajuste do modelo são expostos por Hall Jr. et al. (2011) e adaptados por Ayres Jr. et al. (2014), no ACRP Report 107. A utilização dos modelos 
pressupõe a obtenção de dados operacionais e climatológicos, para que se ajuste a formulação à realidade do aeródromo em estudo. Além disso, disponibiliza-se o software LRSARA para o processamento automatizado dos dados em questão. 0 uso dessa ferramenta requer o acesso a dados operacionais e climáticos relativos ao aeródromo e à sua localização geográfica, otimizando o ajuste do modelo para as condições requeridas.

$$
P\{\text { Ocorrência de Acidentes }\}=\frac{1}{1+e^{b 0+X 1 . b 1+X 2 . b 2+X 3 . b 3} \ldots}
$$

em que: $\quad P$ \{Ocorrência de Acidente\}: $\quad$ probabilidade de um acidente ocorrer dentro das condições operacionais determinadas;

$$
\begin{array}{ll}
X i: & \text { variáveis independentes; e } \\
\text { bi: } & \text { coeficientes de regressão. }
\end{array}
$$

$$
\boldsymbol{P}\{\text { Localização }>\boldsymbol{y}\}=\boldsymbol{e}^{-\boldsymbol{b} \boldsymbol{y}^{\boldsymbol{m}}}
$$

em que: $\quad P\{$ Localização>y\}: $\quad$ probabilidade de a distância de veer-off ser maior que y da extremidade da pista;

$y: \quad$ distância a partir da extremidade da pista; e

b e $m$ : $\quad$ coeficientes de ajuste do modelo.

Para todas as análises, considera-se que outra aeronave está localizada em qualquer segmento da TWY paralela. Esta é uma hipótese conservadora, porque, na maioria dos casos, o obstáculo será uma aeronave em movimento sobre um pequeno trecho da TWY paralela, com um pequeno comprimento em comparação com a extensão total da pista. De forma simplificada e baseada em premissas conservadoras, Hall Jr. et al. (2011) apresentam ábacos para a aplicação direta do método de análise de risco quanto ao veer-off nos procedimentos de pouso e decolagem. 0 método recomenda as seguintes etapas: (i) identificar a RWY e a TWY e o grupo de aeronaves (ADG) a serem avaliados, baseado na aeronave com maior envergadura em operação; (ii) selecionar o gráfico para o ADG específico envolvido na estimativa de risco; e (iii) com base na separação entre os eixos das RWY e TWY, obtém-se o risco associado à operação em tais condições, sendo tal risco aceitável desde que inferior a $1 \times 10^{-7}$.

\section{CONDIÇÕES FÍSICAS E OPERACIONAIS DO AEROPORTO DE ESTUDO}

Segundo ANAC (2018), tendo o Aeroporto Internacional de Fortaleza (SBFZ na nomenclatura ICAO) código de referência 4E, a distância mínima entre eixos de RWY e TWY, em operações com condições meteorológicas por instrumentos (ILS), deve ser de 172,5 m (antes de 2018, era sugerida uma separação mínima de 182,5 m). No referido aeroporto, as distâncias existentes estão abaixo do previsto para o caso de operações com ILS, entretanto, para operações visuais, o projeto geométrico do aeroporto cumpre os requisitos normativos. Esse descumprimento é função de pequenos desvios que fazem com que as TWY não sejam perfeitamente paralelas à RWY 13/31. A Figura 2 apresenta as distâncias entre RWY e TWY para o SBFZ. Neste caso, as distâncias em questão foram obtidas a partir de medições realizadas pelo operador aeroportuário, bem como podem ser conferidas a partir da carta do aeroporto, não havendo neste caso as imprecisões oriundas da medição de distâncias a partir de ferramentas de satélite.

Por estar o Aeroporto Internacional de Fortaleza inserido em área densamente urbanizada, não existe área disponível para a correção dos afastamentos necessários das TWY paralelas Alfa e Juliet, tampouco é viável suspender totalmente o movimento de aeronaves nessas TWY quando a RWY estiver em uso, dado o impacto que seria gerado na capacidade operacional do aeroporto. As TWY Alfa e Juliet, localizadas, respectivamente, ao Sul e ao Norte da RWY, não são 
totalmente paralelas à RWY 13/31. As separações existentes entre as referidas TWY nas suas cabeceiras (Threshold - THR) 13 (ao Oeste) e 31 (ao Leste) são: (i) TWY Alfa - RWY THR $13=$ 162,08 m; (ii) TWY Alfa - RWY THR 31 = 163,47 m; (iii) TWY Juliet - RWY THR 13 = 170,65 m e (iv) TWY Juliet - RWY THR $31=169,04 \mathrm{~m}$. Cumpre mencionar que consta na ANAC um pedido permanentemente deferido de isenção de cumprimento de requisito de infraestrutura para o aeroporto em estudo quanto às distâncias de separação (Decisão no 127/2015, 16/10/2015).

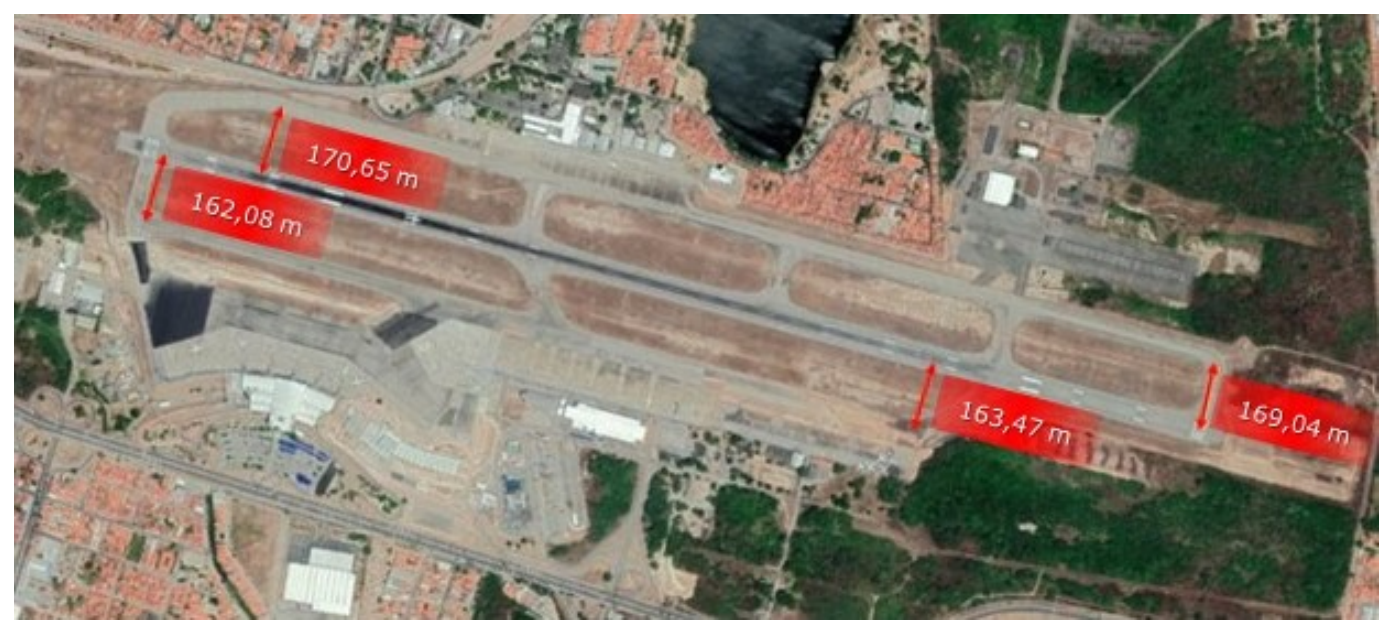

Figura 2. Distâncias (m) entre RWY-TWY paralelas no SBFZ

Uma análise inicial dessas separações parte da conceituação de dois parâmetros: (i) distância entre as pontas de asa das aeronaves: representando, simplesmente, a distância que seria observada no aeródromo em estudo no caso de duas aeronaves operando nas TWY e na RWY paralelas, com a máxima envergadura associada ao código de referência da aeronave e (ii) distância crítica (DC), representando a distância entre as pontas de asa das aeronaves que deveria ser observada caso a separação de 172,5 m fosse devidamente observada. Por exemplo, aeronaves com código E movimentando-se nas RWY/TWY paralelas devem possuir DC de 107,5 m. A partir daí, pode-se avaliar a distância entre pontas de asas quando as separações RWY-TWY são inferiores à regulamentada. De acordo com as operações de aeronaves típicas que ocorrem no Aeroporto Internacional de Fortaleza, comparam-se na Tabela 2 aeronaves de código $\mathrm{C}$ (envergadura maior ou igual a $24 \mathrm{~m}$ e menor que $36 \mathrm{~m}$ ), D (maior ou igual a $36 \mathrm{~m}$ e menor que $52 \mathrm{~m}$ ) e E (envergadura maior ou igual a 52 e menor que $65 \mathrm{~m}$ ). Na Tabela 2, a análise dentro dos limites de 90 e 100\% da DC tem função apenas de ilustrar as combinações mais críticas.

Tabela 2 - Operações com distância de ponta de asa inferiores à Distância Crítica (DC) nas cabeceiras (THR) da RWY do Aeroporto Internacional de Fortaleza

\begin{tabular}{|c|c|c|c|c|c|c|c|c|c|c|c|c|}
\hline \multirow{3}{*}{$\begin{array}{c}\text { Código da Aero- } \\
\text { nave Posicionada } \\
\text { na RWY 13/31 }\end{array}$} & \multicolumn{6}{|c|}{ Taxiway Alfa } & \multicolumn{6}{|c|}{ Taxiway Juliet } \\
\hline & \multicolumn{3}{|c|}{$\begin{array}{c}\text { Aferição Efetuada no } \\
\text { Ponto mais próximo à } \\
\text { THR } 13(\%)\end{array}$} & \multicolumn{3}{|c|}{$\begin{array}{c}\text { Aferição Efetuada no } \\
\text { Ponto mais próximo à } \\
\text { THR } 31 \text { (\%) }\end{array}$} & \multicolumn{3}{|c|}{$\begin{array}{c}\text { Aferição Efetuada no } \\
\text { Ponto mais próximo à } \\
\text { THR } 13(\%)\end{array}$} & \multicolumn{3}{|c|}{$\begin{array}{c}\text { Aferição Efetuada no } \\
\text { Ponto mais próximo à } \\
\text { THR } 31(\%)\end{array}$} \\
\hline & C & D & $\mathbf{E}$ & C & D & $E$ & C & D & $\mathbf{E}$ & C & D & $\mathbf{E}$ \\
\hline C & 107 & 100 & 95 & 108 & 102 & 96 & 115 & 108 & 102 & 113 & 106 & 101 \\
\hline D & 100 & 94 & 89 & 102 & 95 & 89 & 108 & 101 & 95 & 106 & 100 & 94 \\
\hline E & 95 & 88 & 83 & 96 & 89 & 84 & 102 & 95 & 90 & 101 & 94 & 89 \\
\hline * & \multicolumn{12}{|c|}{ Distância de ponta de asa superior a $100 \%$ da DC } \\
\hline$* *$ & \multicolumn{12}{|c|}{ Distância de ponta de asa entre $90 \%$ a $100 \%$ da DC } \\
\hline$* * *$ & \multicolumn{12}{|c|}{ Distância de ponta de asa inferior a $90 \%$ da DC } \\
\hline
\end{tabular}


Verifica-se, com base na Tabela 2, que as situações de maior comprometimento para a segurança das operações na RWY e TWY do Aeroporto Internacional de Fortaleza envolvem as aeronaves de maior porte (códigos D e E), na TWY Alfa. Cabe ainda informar que o Aeroporto Internacional de Fortaleza se localiza a $25 \mathrm{~m}$ de altitude em relação ao nível do mar e apresenta condições meteorológicas constantes durante a maior parte do ano, fator também favorável às operações aéreas. Sabe-se que as condições meteorológicas relacionadas com vento, teto e visibilidade são as que mais contribuem para os acidentes aeronáuticos que têm o fator meteorologia como principal causa.

Para a operação em condições visuais, a condição de visibilidade mínima deve ser de 1.500 m, o que ocorreu em cerca de $98 \%$ do tempo no aeroporto em estudo. A condição de teto foi superior a $450 \mathrm{~m}$ em mais de $97 \%$ do tempo, considerando os dados do ano de 2018. A direção de ventos constantes possibilitou uma média de 99,83\% das operações pela THR 13 e de 0,17\% pela THR 31, no período de 2010 a 2018. Assim como ocorre em todo o estado do Ceará, as precipitações sobre o aeródromo são irregulares e sazonais, concentradas no primeiro semestre do ano. De 2010 a 2018, a média de tempo com operações em condições meteorológicas visuais foi de 98,38\%, enquanto que em condições instrumentadas foi de 1,62\%, o que corrobora com os resultados de visibilidade e teto apresentados anteriormente (REDEMET, 2018). A partir da aplicação dos coeficientes expostos em Ayres Jr. et al. (2014), dispõe-se de um conjunto de dados que permite uma aplicação mais completa do modelo matemático mostrado nas Equações 1 e 2, ressalvando-se que maior precisão seria obtida quanto mais completos fossem os dados.

\section{RESULTADOS E DISCUSSÃO}

\subsection{Abordagem simplificada (método gráfico)}

As Tabelas 3 a 5 apresentam as avaliações de risco de colisão para a aeronave mais crítica em operação (Categoria E) no Aeroporto Internacional de Fortaleza (SBFZ na nomenclatura ICAO), e na maioria dos aeroportos internacionais no território nacional, para as situações de: (i) aproximação perdida no pouso, (ii) veer-off em corrida de pouso e (iii) veer-off em corrida de decolagem, para as distâncias de separação entre a RWY 13/31 e as TWY Alfa e Juliet. 0 procedimento mostrado foi similar às demais categorias avaliadas neste trabalho (C/D). Convém mencionar que os resultados expostos carregam em si a imprecisão inerente a qualquer interpretação em métodos gráficos, que podem variar de acordo com o rigor do observador, por exemplo. No caso do Aeroporto Internacional de Fortaleza, as imprecisões que porventura estejam embutidas nessa análise não a comprometem, especialmente pelo fato de que em todos os casos, para as separações entre pistas existentes, são obtidos riscos inferiores ao limite de $10^{-7}$.

De forma análoga, foram feitas as análises referentes às aeronaves de categoria $\mathrm{C} \mathrm{e} \mathrm{D.} \mathrm{Deve-}$ se observar que os códigos de referência C, D e E representam, respectivamente, os códigos ADG III, IV e V. Em resumo, a análise de dados obtidos a partir dos ábacos presentes no documento do método proposto resulta em um conjunto de resultados mostrado na Tabela 6.

Os dados mostrados na Tabela 6 representam riscos de colisão que variam da ordem de uma ocorrência a cada 31 milhões de pousos a uma ocorrência em mais de 1 bilhão de pousos. Observados em perspectiva temporal futura quanto ao fluxo operacional de aeronaves no Aeroporto Internacional de Fortaleza no ano de 2018, observa-se que esses riscos seriam proporcionais a um período de tempo muito maior do que o previsto para uma futura desativação do sítio aeroportuário. Verifica-se, assim, que, em todas as estimativas, foram obtidos valores de 
risco inferiores a $10^{-7}$ (ou seja, menos de uma ocorrência para cada 10 milhões de operações), considerados aceitáveis para o risco de colisão durante as operações, indicando que as condições de operação do aeroporto em estudo são seguras.

Tabela 3 - Risco de colisão - Aproximação perdida no pouso (Categoria E, TWY Alfa e Juliet)

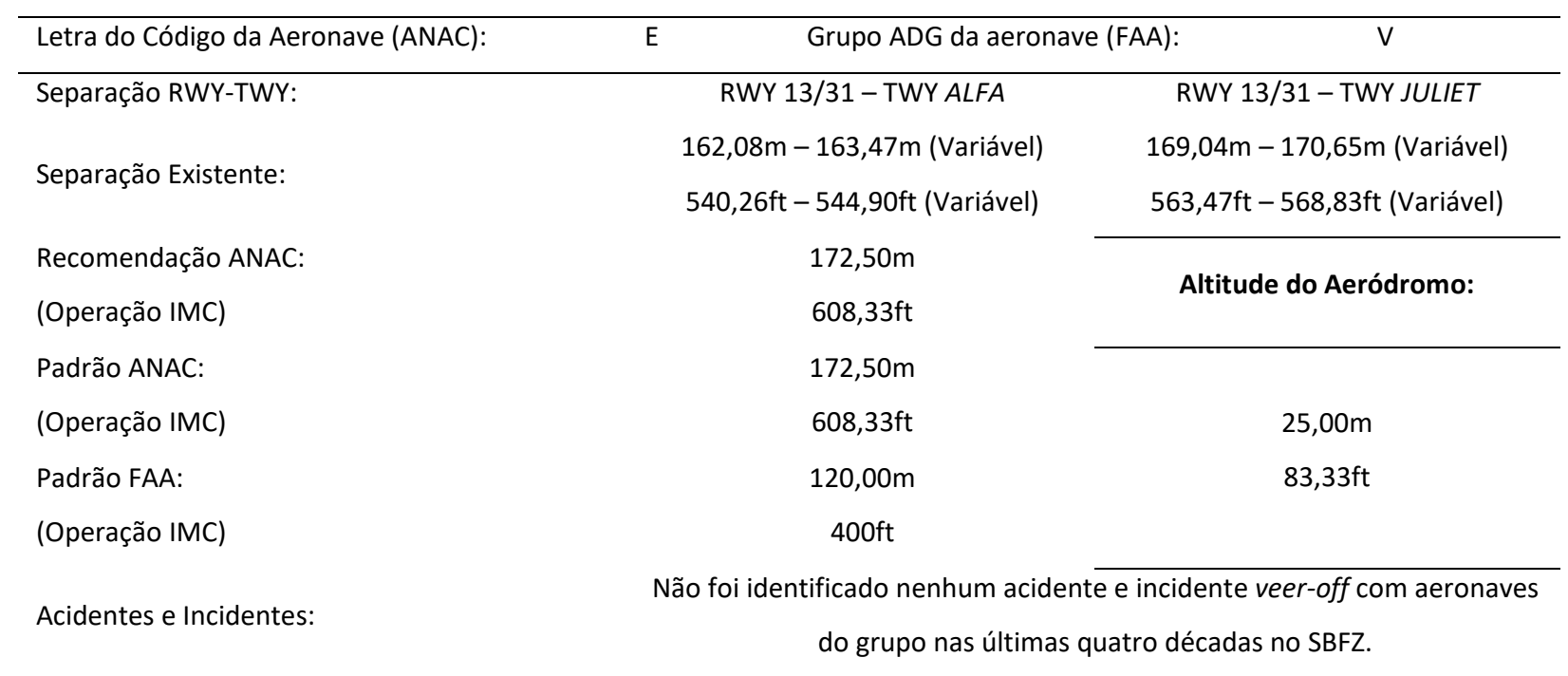

Cálculo do Risco de Colisão: POUSO - APROXIMAÇÃo PERDIDA (Fonte: Hall Jr et al., 2011)

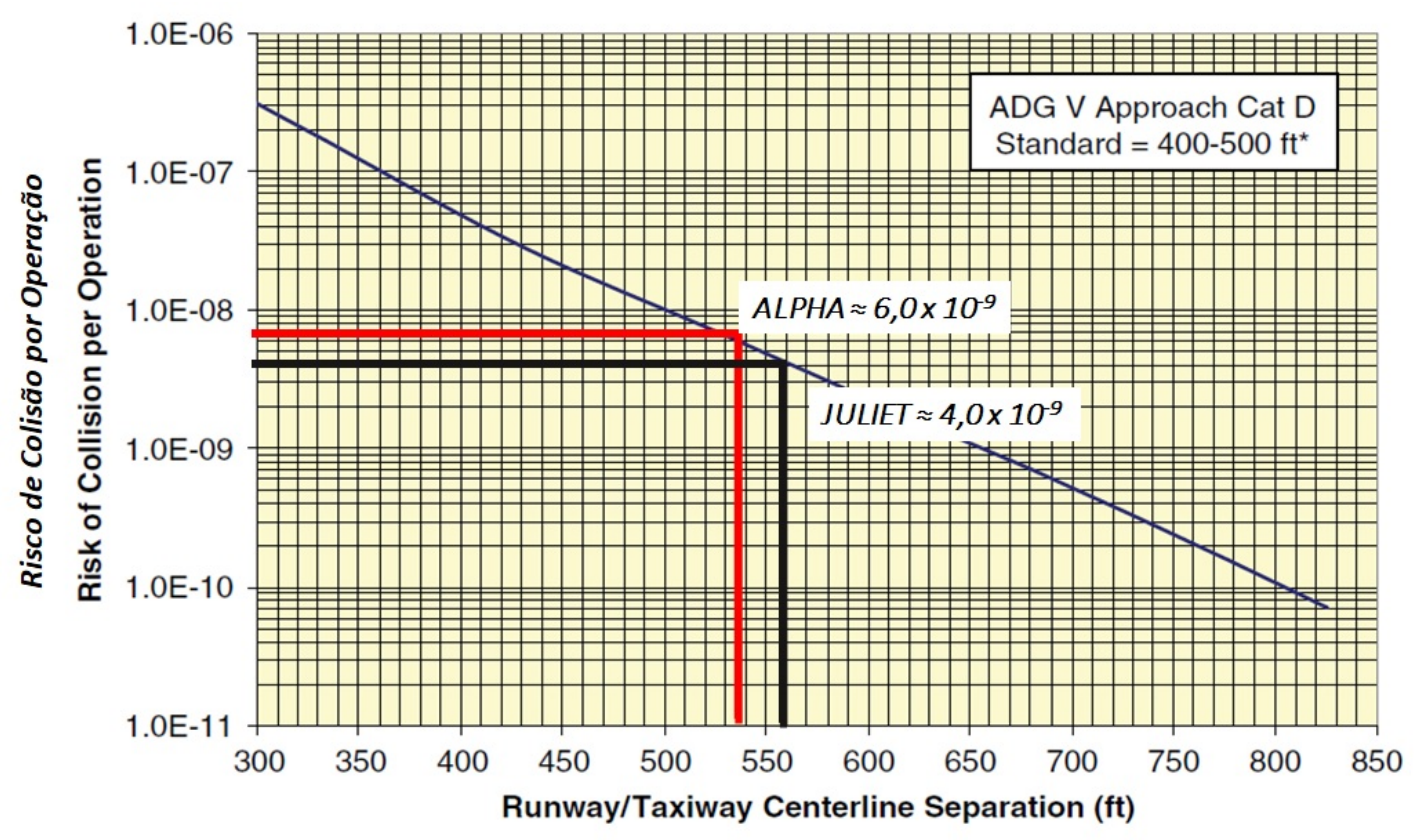

Separação RWY/TWY (pés)

\begin{tabular}{llll}
\hline Nível de Risco 1: & $\approx 6,0 \times 10^{-9}$ & Resultado: & Aceitável \\
ALFA & $\approx 4,0 \times 10^{-9}$ & Resultado: & Aceitável \\
\hline Nível de Risco 2: & & \\
JULIET & & \\
\hline
\end{tabular}


Tabela 4 - Risco de colisão - Veer-off em corrida de pouso (Categoria E, TWY Alfa e Juliet)

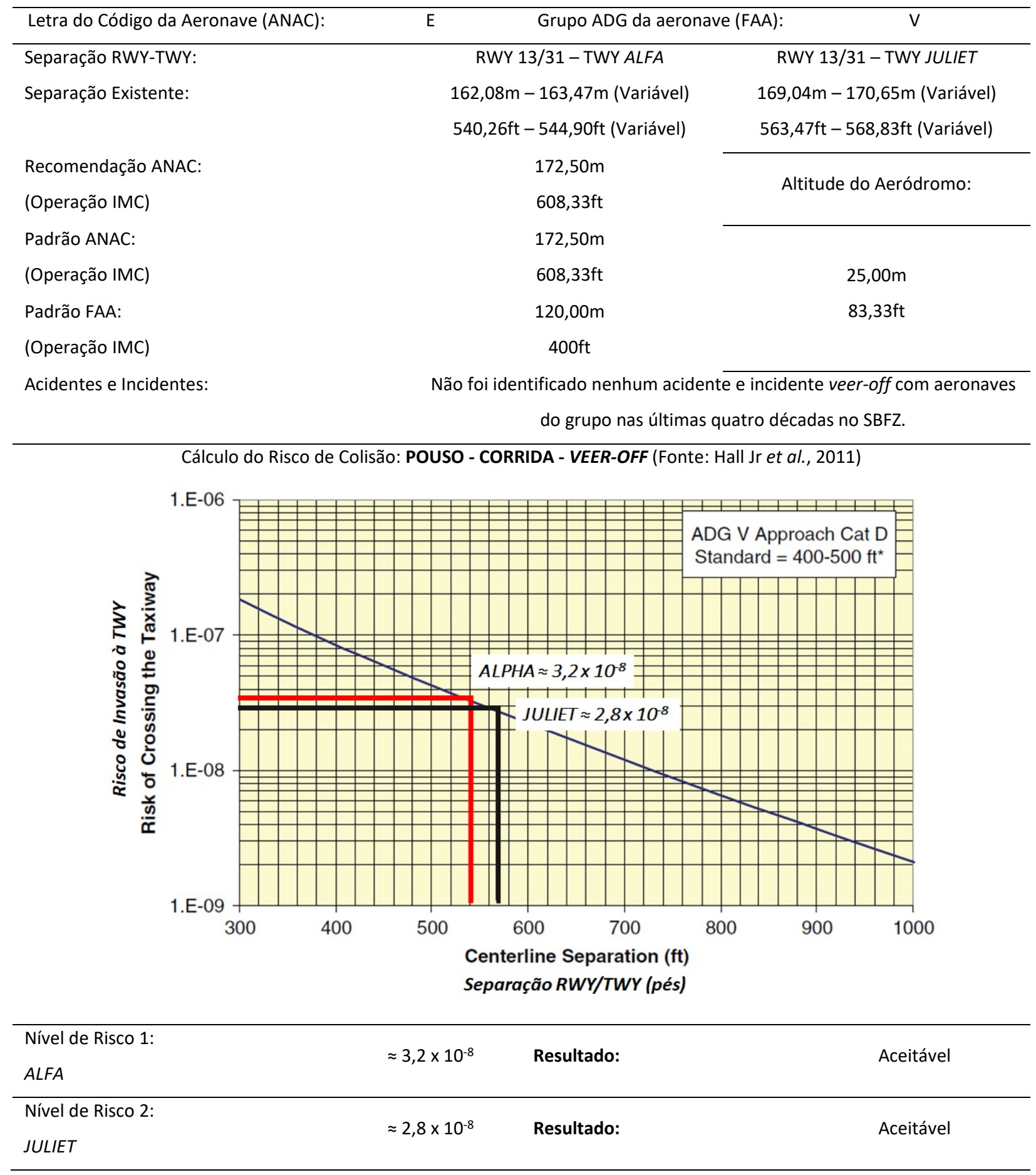


Tabela 5 - Risco de colisão - Veer-off em corrida de decolagem (Categoria E, TWY Alfa e Juliet)

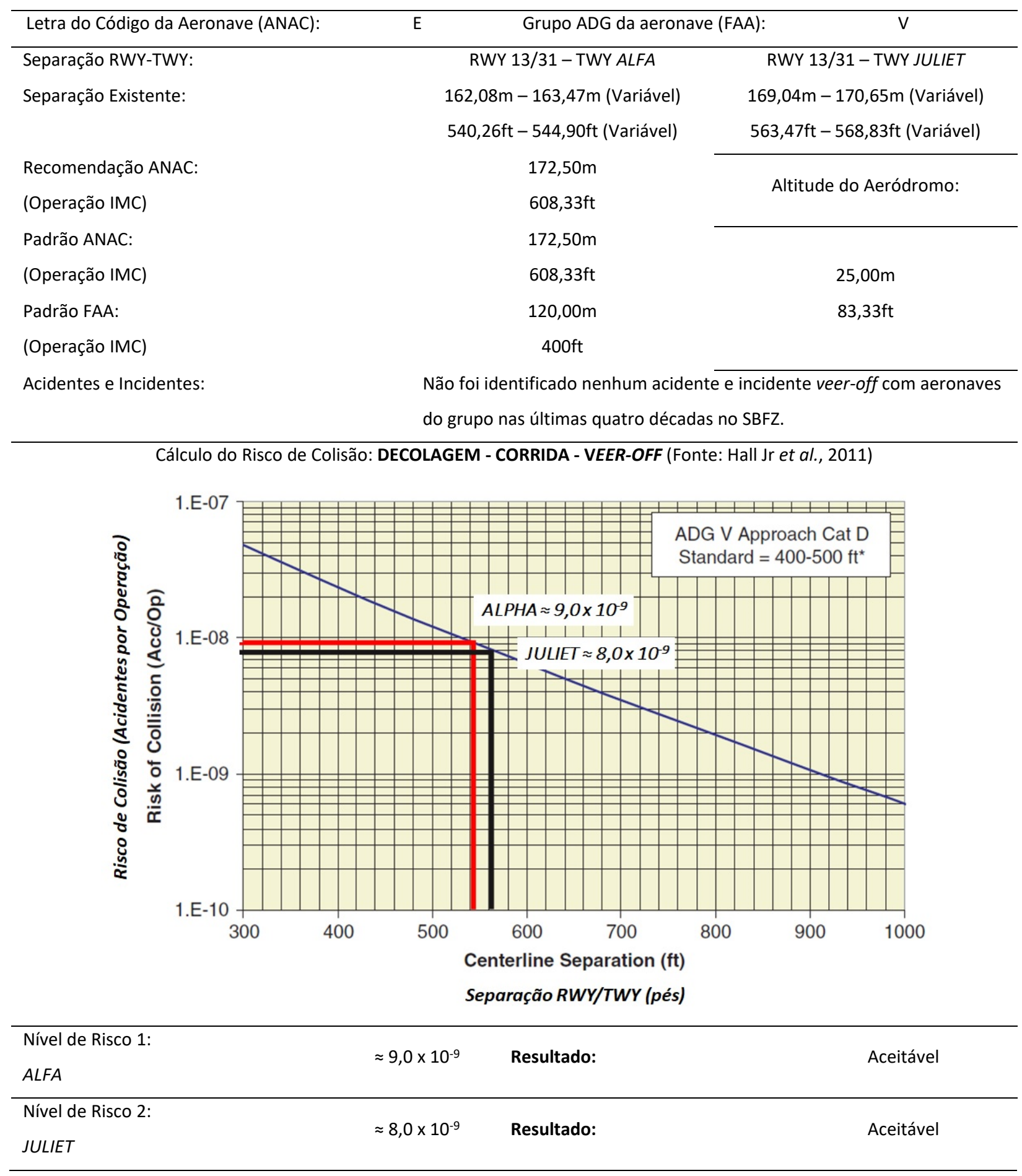


Tabela 6 - Estimativas risco de colisão entre aeronaves utilizando simultaneamente a RWY 13/31 e as TWY Alfa e Juliet

\begin{tabular}{cccccc}
\hline \multirow{2}{*}{$\begin{array}{c}\text { Código da } \\
\text { Aeronave } \\
\text { (ANAC) }\end{array}$} & $\begin{array}{c}\text { Grupo ADG da } \\
\text { Aeronave (FAA) }\end{array}$ & $\begin{array}{c}\text { TWY } \\
\text { Paralela }\end{array}$ & $\begin{array}{c}\text { Aproximação } \\
\text { Perdida no Pouso }\end{array}$ & $\begin{array}{c}\text { Veer-off em Corrida } \\
\text { de Pouso }\end{array}$ & $\begin{array}{c}\text { Veer-off em Corrida } \\
\text { de Decolagem }\end{array}$ \\
\cline { 4 - 6 } C & III & Alfa & $<8,0 \times 10^{-11}$ & $\approx 2,0 \times 10^{-8}$ & $\approx 6,0 \times 10^{-9}$ \\
D & Juliet & $<8,0 \times 10^{-11}$ & $\approx 1,8 \times 10^{-8}$ & $\approx 5,0 \times 10^{-9}$ \\
IV & Alfa & $\approx 2,0 \times 10^{-9}$ & $\approx 2,8 \times 10^{-8}$ & $\approx 8,0 \times 10^{-9}$ \\
E & Juliet & $\approx 1,5 \times 10^{-9}$ & $\approx 2,6 \times 10^{-8}$ & $\approx 6,8 \times 10^{-9}$ \\
& Alfa & $\approx 6,0 \times 10^{-9}$ & $\approx 3,2 \times 10^{-8}$ & $\approx 9,0 \times 10^{-9}$ \\
& Juliet & $\approx 4,0 \times 10^{-9}$ & $\approx 2,8 \times 10^{-8}$ & $\approx 8,0 \times 10^{-9}$ \\
\hline
\end{tabular}

\subsection{Aplicação do modelo matemático proposto nos ACRP Reports $051 / 107$}

A aplicação do modelo matemático em questão prevê, inicialmente a definição de algumas premissas, que tratam de adaptar o modelo à realidade do sítio aeroportuário em estudo. Essas premissas refletem na adoção dos coeficientes de regressão (b) a serem aplicados na Equação 2, anteriormente exposta. A partir das condições expostas ao longo do item 5 para a seleção dos referidos coeficientes, contidos em Ayres Jr. et al. (2014), resultou-se na análise de riscos mostrada na Tabela 7.

Tabela 7 - Estimativas risco de colisão entre aeronaves utilizando simultaneamente a RWY 13/31 e as TWY Alfa e Juliet

\begin{tabular}{|c|c|c|c|c|c|c|c|c|c|c|c|c|c|}
\hline & & \multicolumn{6}{|c|}{ Taxiway Alfa } & \multicolumn{6}{|c|}{ Taxiway Juliet } \\
\hline & & \multicolumn{3}{|c|}{$\begin{array}{c}\text { Aferição Efetuada no } \\
\text { Ponto mais próximo à } \\
\text { THR } 13(\%)\end{array}$} & \multicolumn{3}{|c|}{$\begin{array}{c}\text { Aferição Efetuada no } \\
\text { Ponto mais próximo à } \\
\text { THR } 31(\%)\end{array}$} & \multicolumn{3}{|c|}{$\begin{array}{c}\text { Aferição Efetuada no } \\
\text { Ponto mais próximo à } \\
\text { THR } 13(\%)\end{array}$} & \multicolumn{3}{|c|}{$\begin{array}{c}\text { Aferição Efetuada no } \\
\text { Ponto mais próximo à } \\
\text { THR } 31(\%)\end{array}$} \\
\hline & & $\mathrm{C}$ & D & $\mathrm{E}$ & $\mathrm{C}$ & D & $E$ & C & D & $E$ & C & D & $E$ \\
\hline \multirow{3}{*}{ Pouso } & $\mathrm{C}$ & $2 \mathrm{E}-08$ & $2 \mathrm{E}-08$ & $3 \mathrm{E}-08$ & $2 \mathrm{E}-08$ & $2 \mathrm{E}-08$ & $2 \mathrm{E}-08$ & $2 \mathrm{E}-08$ & $2 \mathrm{E}-08$ & $2 \mathrm{E}-08$ & $2 \mathrm{E}-08$ & $2 \mathrm{E}-08$ & $2 \mathrm{E}-08$ \\
\hline & D & $2 \mathrm{E}-08$ & $3 \mathrm{E}-08$ & $3 \mathrm{E}-08$ & $2 \mathrm{E}-08$ & $3 \mathrm{E}-08$ & $3 \mathrm{E}-08$ & $2 \mathrm{E}-08$ & $2 \mathrm{E}-08$ & $2 \mathrm{E}-08$ & $2 \mathrm{E}-08$ & $2 \mathrm{E}-08$ & $3 \mathrm{E}-08$ \\
\hline & $\mathbf{E}$ & $3 \mathrm{E}-08$ & $3 \mathrm{E}-08$ & $3 \mathrm{E}-08$ & $2 \mathrm{E}-08$ & $3 \mathrm{E}-08$ & $3 \mathrm{E}-08$ & $2 \mathrm{E}-08$ & $2 \mathrm{E}-08$ & $3 \mathrm{E}-08$ & $2 \mathrm{E}-08$ & $3 \mathrm{E}-08$ & $3 \mathrm{E}-08$ \\
\hline \multirow{3}{*}{ Decolagem } & C & $6 \mathrm{E}-11$ & $7 E-11$ & $8 \mathrm{E}-11$ & $6 \mathrm{E}-11$ & $7 \mathrm{E}-11$ & $8 \mathrm{E}-11$ & $5 E-11$ & $6 \mathrm{E}-11$ & $7 \mathrm{E}-11$ & $6 \mathrm{E}-11$ & $7 \mathrm{E}-11$ & 7E-11 \\
\hline & D & $7 \mathrm{E}-11$ & $9 \mathrm{E}-11$ & $1 \mathrm{E}-10$ & $7 \mathrm{E}-11$ & $8 \mathrm{E}-11$ & $1 \mathrm{E}-10$ & $6 \mathrm{E}-11$ & $7 \mathrm{E}-11$ & $8 \mathrm{E}-11$ & $7 \mathrm{E}-11$ & $8 \mathrm{E}-11$ & $9 \mathrm{E}-11$ \\
\hline & $\mathbf{E}$ & $8 \mathrm{E}-11$ & $1 \mathrm{E}-10$ & $1 \mathrm{E}-10$ & $8 \mathrm{E}-11$ & $1 \mathrm{E}-10$ & $1 \mathrm{E}-10$ & $7 \mathrm{E}-11$ & $8 \mathrm{E}-11$ & $9 \mathrm{E}-11$ & $7 \mathrm{E}-11$ & $9 \mathrm{E}-11$ & $1 \mathrm{E}-10$ \\
\hline
\end{tabular}

Convém mencionar que a aplicação desse modelo partiu de algumas premissas simplificadoras, como a utilização somente de aeronaves de grande porte, ventos perpendiculares entre $2 \mathrm{e}$ 5 nós, vento de cauda entre 5 e 12 nós, condições ideais de visibilidade na maior parte do tempo, temperatura acima de $25^{\circ} \mathrm{C}$, bem como a presença constante de chuva, sem nenhuma ocorrência de eventos como neve, congelamento ou eventos mais diretamente associados a zonas temperadas. Além disso, cabe também a menção ao fato de que o Aeroporto Internacional de Fortaleza possui considerável número de voos internacionais, sendo um hub na ligação Brasil-Europa/EUA. No modelo de localização, aplicaram-se as distâncias em relação ao limiar da pista para que a distância de ponta de asa fosse igual a zero, dependendo, exclusivamente, da categoria da aeronave operando na pista (C/D/E) ou nas taxiways. Na tabela 7 se observa que essas condições levam à um nível de risco, especialmente em relação à decolagem, 100 vezes menor, 
enquanto que para a corrida de pouso, os riscos apresentam a mesma ordem de grandeza observada na Tabela 6, produzida com a aplicação de ábacos. Um dos principais pontos positivos da aplicação do modelo matemático em detrimento ao método gráfico é a possibilidade de se avaliar o risco para aeronaves de diferentes categorias operando nas RWY e TWY paralelas.

\section{CONCLUSÕES}

No Brasil, alguns aeroportos apresentam inconformidades de separações entre eixos de pistas de pouso e decolagem e de taxiamento paralelas que são inferiores aos requisitos exigidos pela ANAC. Para que se verifique o nível de segurança de operações sob tais condições, foi aplicado neste trabalho o método de análise de risco desenvolvido pelo Airport Cooperative Research Program do Transportation Research Board. Esse método apresentou-se como um meio formal e adequado para análises de probabilidade de colisão na faixa de pista durante as operações de pouso e decolagem com aplicação para aeroportos brasileiros. Dentre os benefícios da sua utilização está a melhoria e a adequação dos projetos geométricos de aeródromos e a análise da segurança operacional, além da possibilidade do projetista evitar ou quantificar a tendência de colisão entre aeronaves em relação a obstáculos situados no aeródromo, com base na distância entre as pontas de asa das aeronaves posicionadas na pista de pouso e decolagem (RWY) e na pista de taxiamento (TWY) paralela.

Destaca-se que o método permite a otimização operacional quando aeronaves de classes diferentes estiverem utilizando RWY e TWY paralelas, servindo como uma ferramenta eficiente para os operadores aeroportuários eliminarem ou reduzirem os riscos quando em operações simultâneas de aeronaves. Pode-se afirmar que o método fundamenta as tomadas de decisões viáveis para projetos no lado ar de aeródromos e fomenta a análise de capacidade do nível de segurança operacional existente em situações de inconformidades. Verificou-se que a abordagem simplificada do método levou a resultados similares aos obtidos para o modelo customizado para o aeródromo específico em estudo, especialmente quanto à corrida de pouso, mostrando-se que ambas as abordagens são válidas.

Quanto ao Aeroporto Internacional de Fortaleza, as distâncias de separação existentes no cenário atual são inferiores ao previsto nos regulamentos brasileiros para aeródromos de código de referência $4 \mathrm{E}$, operando por instrumentos, porém superiores às distâncias previstas para aeródromos semelhantes que operem apenas em condições visuais. Concluiu-se que, no mencionado aeroporto, os riscos associados a colisões em função da inconformidade de separações de RWY-TWY paralelas são, em geral, de uma ocorrência em uma ordem de milhões de operações. Isso permite ao operador do aeródromo um razoável grau de confiança na segurança operacional do aeródromo, considerando sua demanda ao longo do tempo.

Espera-se que as análises expostas neste artigo contribuam para um incremento da segurança operacional dos aeródromos do território nacional, além de guiarem a uma melhoria na concepção de projetos de RWY e TWY e às adequações dessas infraestruturas em aeroportos brasileiros mais antigos.

\section{REFERÊNCIAS}

ANAC (2012). Regulamento Brasileiro da Aviação Civil - RBAC nำ154, Emenda no 01: Projeto de Aeródromos. Secretaria da Aviação Civil, Brasília, DF.

ANAC (2015). Regulamento Brasileiro da Aviação Civil - RBAC nº139, Emenda no 05: Certificação Operacional de Aeroportos. Secretaria da Aviação Civil, Brasília, DF.

ANAC (2018). Regulamento Brasileiro da Aviação Civil - RBAC nº154, Emenda no 03: Projeto de Aeródromos. Secretaria da Aviação Civil, Brasília, DF. 
Ayres Jr., M.; Shirazi, H.; Carvalho, R.; Hall Jr., J. W.; Speir, R.; Arambula, E. David, R.; Gadzinski, J.; Caves, R.; Wong, D.; Pitfield, D. (2013) Modelling the location and consequences of aircraft accidents. Safety Science, V. 51, 178-186. DOI: https://doi.org/10.1016/j.ssci.2012.05.012.

Ayres Jr, M.; Carvalho, R.; Shirazi, H.; David, R. E. (2014) ACRP Report 107 - Development of a runway veer-off location distribution risk assessment model and reporting template. Transportation Research Board (TRB), Washington-DC, EUA.

FAA (2012). Advisory Circular AC no 150/5300-13A: Airport Design. Federal Aviation Administration, U.S. Department of Transportation, EUA.

Fernandes, H. F.; Müller, C.; Alves, C. J. P. (2018) Avaliação dos requisitos da faixa de pista em aeroportos regionais: uma análise de risco. Revista Transportes, v. 26, p 76-91. DOI: 10.14295/transportes.v26i4.1478

Hall Jr., J.; Ayres Jr., M.; Wong, D.; Appleyard, A.; Eddowes, M.; Speir, R.; Pitfield, D.; Caves, R.; Selezneva, O.; Puzin, T. (2008) ACRP Report 3 - Analysis of aircraft overruns and undershoots for runway safety areas. Transportation Research Board (TRB), Washington, D.C., EUA.

Hall Jr., J.; Ayres Jr., M.; Shirazi, H.; Speir, R.; Carvalho, R.; David, R.; Huang, Y. (2011) ACRP Report 51 - Risk assessment method to support modification of airfield separation standards. Transportation Research Board (TRB), Washington-DC, EUA.

Huang Z. (2018) Sensitivity Analysis of Runway Veer-off Risk Assessment. Master of Science in Civil Engineering for Risk Mitigation, School of Civil, Environmental and Land Management Engineering, Politecnico di Milano, Milão, Itália.

ICAO (2018) Annex 14 to the Convention on International Civil Aviation - Aerodrome Design and Operations. 8th Edition. International Civil Aviation Organization. Montreal, Canadá.

Ketabdari, M.; Giustozzi, F.; Crispino, M. (2017) Sensitivity analysis of influencing factors in probabilistic risk assessment for airports. Safety Science. DOI: http://dx.doi.org/10.1016/j.ssci.2017.07.005

REDEMET (2018) Meteorological Aerodrome Report - Aeroporto Internacional de Fortaleza - 2018. Rede Meteorologia do Comando da Aeronáutica. <https://www.redemet.aer.mil.br/?i=produtos\&p=consulta-de-mensagens-opmet $>$ (dezembro, 2018).

Travers, P. C. (1995) New large aircraft. Institution of Mechanical Engineers Journal, V. 209. Birmingham, Reino Unido. 